\title{
Klebsiella pneumoniae presents antimicrobial drug resistance for $\beta$-lactam through the ESBL/PBP signaling pathway
}

\author{
WEI JIANG ${ }^{1}$, WENJIE YANG $^{2}$, XUEQUN ZHAO $^{2}$, NA WANG $^{1}$ and HAIXIA REN ${ }^{3}$ \\ Departments of ${ }^{1}$ Infectious Disease, ${ }^{2}$ Transplantation and ${ }^{3}$ Pharmaceutical Preparation, \\ Tianjin First Central Hospital, Tianjin 300000, P.R. China
}

Received August 13, 2018; Accepted November 25, 2019

DOI: $10.3892 /$ etm. 2020.8498

\begin{abstract}
Overuse and misuse of antibiotics leads to antibiotic resistance which has become a significant public health concern. Klebsiella pneumoniae is the most common pathogenic bacteria underlying nosocomial infections due to the expression of virulence factors and occurrence of antibiotic resistance. Evidence indicates that $\beta$-lactamase is involved in the antibiotic resistance of Klebsiella pneumoniae to $\beta$-lactam antibiotics. The aim of the present study was to investigate the association between the molecular biological mechanisms of antibiotic resistance of Klebsiella pneumoniae and extended-spectrum $\beta$-lactamase (ESBL). In order to assess temporal trends in prevalence and antimicrobial susceptibility, Klebsiella pneumoniae bacteria were isolated and the ESBL expression level was analyzed. Susceptibility tests were performed using automated systems. The $\beta$-lactam-resistance in Klebsiella pneumoniae was assessed by the $\beta$-lactam agar screen plate and respective MIC values were evaluated using E-test strips. The confirmatory disk diffusion methods were applied for phenotypic identification of the ESBL production of Klebsiella pneumoniae. The results showed that Klebsiella pneumoniae bacteria exhibited higher ESBL production after treatment with $\beta$-lactam compared to the control. The ESBL gene expression was upregulated in Klebsiella pneumoniae after treatment with $\beta$-lactam. Results identified that penicillin-binding proteins (PBPs) were associated with the growth and resistance to $\beta$-lactams. Zinc finger nuclease markedly inhibited the antibiotic resistance of Klebsiella pneumoniae to $\beta$-lactam. PBP knockdown abolished the inhibitory effects of zinc finger nuclease on the growth of Klebsiella pneumoniae induced by $\beta$-lactam antibiotic treatment. In conclusion, these results suggest that the resistance of Klebsiella pneumoniae bacteria to antimicrobial drugs
\end{abstract}

Correspondence to: Professor Wei Jiang, Department of Infectious Disease, Tianjin First Central Hospital, 24 Dukang Road, Tianjin 300000, P.R. China

E-mail: weijiangsteel@163.com

Key words: Klebsiella pneumoniae, $\beta$-lactam, antibiotic resistance, extended-spectrum $\beta$-lactamase, penicillin-binding proteins, zinc finger nuclease is through the ESBL signaling pathway, which indicates that ESBL may be a potential target for abolishing resistance to $\beta$-lactam.

\section{Introduction}

In the past several decades, overuse and misuse of antibiotics has led to increased antibiotic resistance which has become a significant public health concern (1). There is no systematic surveillance of antibiotic misuse and no data are available to quantify the problem $(2,3)$. Currently, antibiotic resistance presents an increasing trend that leads to significant public health issues and the increase in healthcare-associated infections caused by antibiotic-resistant pathogens worldwide $(4,5)$. Resistance to different antimicrobial drugs is attributed to the increased irregular employment of antibiotics that have resulted in the drug resistance of expression of virulence factors, promotion of intra-hospital cross infection and even increased challenges of clinical treatments (6-8). A previous report suggests that the major driving force for the occurrence of antibiotic-resistant pathogens is the evolution of metabolic function caused by the rapid antibiotic consumption worldwide (9). Therefore, on the one hand, health organizations are required to implement appropriate policies to supervise antibiotic usage to reduce antibiotic resistance. On the other hand, understanding the molecular biological mechanisms underlying antimicrobial resistance is imminent to evaluate the effects of intervention strategies.

Currently, antimicrobial resistance of gram-negative bacilli is a persistent issue (10). Antibiotic treatment is the most common therapeutic regiment for pulmonary infections with Klebsiella pneumoniae in patients with pneumonia (11). The current antimicrobial resistance and susceptibility of Klebsiella pneumoniae bacteria have been observed in clinical practice (12). Previous research has shown that the frequent outbreak of nosocomial infections is due to extended-spectrum $\beta$-lactamase (ESBL) produced by Klebsiella pneumoniae that is attributed to multiple mechanisms underlying drug resistance (13). In addition, strains of Klebsiella pneumoniae exhibit transferable multiple drug resistance based on clinical sepsis observation (14). Furthermore, antibacterial drug susceptibility of Klebsiella pneumoniae has attracted attention since pathogenic bacteria have acquired simultaneous resistance to various antimicrobial classes mediated by the 
production of ESBL. However, no precise molecular biological mechanisms underlying the antimicrobial drug resistance of Klebsiella pneumoniae have been reported (15).

The correlation between antimicrobial drug resistance and biofilm formation along with ESBL lactamase produced in Escherichia coli has been demonstrated in a previous study (16). Recently, the increase in drug resistance among Klebsiella pneumoniae has caused a great problem in the treatment of pneumonia (17). The mechanisms involved when $\beta$-lactamase hydrolyzes $\beta$-lactam antibiotics have been investigated by performing different experiments (18). Previous research indicates that ancient evolutionary relationships between $\beta$-lactamases and antibiotic-producing bacteria are relatively conservative (19). In any way, antibiotic-resistance genes originate in antibiotic-producing microorganisms and subsequently integrate into the genome of other pathogens through transduction and/or transformation $(20,21)$. Research has found that penicillin-binding proteins (PBPs), membrane-associated macromolecules, play key roles in the cell wall synthesis process (22). In addition, zinc finger nuclease is a new approach to overcome $\beta$-lactam antibiotic resistance (23).

In the present study, it was hypothesized that interfering with ESBL synthesis could decrease antimicrobial drug resistance leading to the control of nosocomial infections, transmission and cross infection. The study also investigated the association between the molecular biological mechanism underlying the antibiotic resistance of Klebsiella pneumoniae and the ESBL/PBP signaling pathway. The present study was designed to elucidate $\beta$-lactam resistance and to understand the efficacy of PBPs and zinc finger nuclease in increasing ESBL expression.

\section{Materials and methods}

Klebsiella pneumoniae culture and reagents. Natural being Klebsiella pneumoniae (NB-K.p) bacteria were purchased from American Type Culture Collection (Klebsiella pneumoniae subsp. Pneumoniae; $\left.\mathrm{ATCC}^{\circledR} 43816^{\mathrm{TM}}\right)$. Klebsiella pneumoniae bacteria from patients with pneumonia (PD-K.p) were isolated from a 56-year male patient with pneumonia who suffered from the disease for approximately 30 years. Cells of Klebsiella pneumoniae were grown in LBmedium at $37^{\circ} \mathrm{C}$ for $24 \mathrm{~h}$.

Growth potential assay. The Klebsiella pneumoniae bacteria were cultured in $10 \mathrm{mg} / \mathrm{ml}$ penicillin medium with or without penicillin-binding proteins (PBPs, $0.67 \mu \mathrm{g} / \mathrm{ml}$, Sigma-Aldrich; Merck KGaA) for $24 \mathrm{~h}$. The number of Klebsiella pneumoniae cells was calculated in the agar plating. The detailed procedures were conducted according to a previous study (24).

Plasmid construction. To investigate the site of the zinc finger nuclease, a recombinant plasmid expressing GFP and ZFN (rpGFP-ZFN) was constructed. All plasmids were purchased from Invitrogen (Thermo Fisher Scientific, Inc.). A full-length ZFN fragment was amplified and subcloned into rpGFP-pET27b. The recombinant plasmid was named rpGFP-ZFN. All expression plasmids were confirmed by sequencing. Cells were transfected with rpGFP-ZFN or
pET27b by using electrotransfection according to the manufacturer's instructions. After a 48-h transfection, the cells were captured using a Leica DM5000 microscope equipped with Q-Imaging Retiga 4000RV camera (Teledyne QImaging).

Antimicrobial susceptibility testing. Antimicrobial susceptibility tests of Klebsiella pneumoniae were performed by the disk diffusion method, according to Clinical and Laboratory Standards Institute (CLSI) recommendations (25). The final results were performed according to the respective standards for antimicrobial susceptibility testing.

Enzyme-linked immunosorbent assay (ELISA). This study analyzed the affinity of PBP with penicillin by using ELISA Kit (cat. no. B21210; R\&D Systems). The ELISA assays were performed according to the manufacturer's protocols. The results were measured at $450 \mathrm{~nm}$ in an ELISA reader.

Real-time quantitative PCR (RT-qPCR) analysis. Total RNA was obtained from Klebsiella pneumoniae by using RNeasy Mini Kit (Qiagen) according to the manufacturer's instructions. The cDNA was synthesized with ReverTraAce (Toyobo Corp.). All forward and reverse primers were synthesized by Invitrogen (Thermo Fisher Scientific, Inc.) and are listed in Table I. PCR amplification was followed by preliminary denaturation at $94^{\circ} \mathrm{C}$ for $2 \mathrm{~min}$, followed by 45 cycles of $95^{\circ} \mathrm{C}$ for $30 \mathrm{sec}$; annealing temperature was reduced to $57^{\circ} \mathrm{C}$ for $30 \mathrm{sec}$, and $72^{\circ} \mathrm{C}$ for $30 \mathrm{sec}$. A volume of $20 \mu \mathrm{l}$ containing $50 \mathrm{ng}$ of genomic DNA, $200 \mu \mathrm{M}$ dNTP, 2.5 units of Taq DNA polymerase, and $200 \mu \mathrm{M}$ primers was used. Relative mRNA expression level changes were calculated using the $2^{-\Delta \Delta \mathrm{Ca}}$ method (25). The results are expressed as $n$-fold compared with the $\beta$-actin control.

Western blot analysis. Klebsiella pneumonia cells were homogenized using RIPA buffer (M-PER reagent for the cells and T-PER reagent for the tissues; Thermo Fisher Scientific, Inc.) and centrifuged at $6,000 \mathrm{xg}$ at $4^{\circ} \mathrm{C}$ for $15 \mathrm{~min}$. The protein concentration was measured using a BCA protein assay kit (Thermo Fisher Scientific, Inc.). A total of $30 \mu \mathrm{g}$ protein extracts was electrophoresed on $12 \%$ polyacrylamide gradient gels and then transferred to polyvinylidene fluoride (PVDF) membrane. The membranes were incubated in 5\% BSA (Sigma-Aldrich; Merck $\mathrm{KGaA}$ ) for $2 \mathrm{~h}$ at $37^{\circ} \mathrm{C}$ and then incubated with primary antibodies (dilution 1:1,000, cat. no. ab11251; Abcam) or $\beta$-actin (dilution 1:1,000, cat. no. ab8226; Abcam) for $2 \mathrm{~h}$ at $37^{\circ} \mathrm{C}$. After washing with PBS three times, the membranes were then incubating with secondary antibodies (dilution 1:1,000, cat. no. SAB11045182; Sigma-Aldrich; Merck KGaA) for $12 \mathrm{~h}$ at $4^{\circ} \mathrm{C}$. After three washings in PBST, the membranes were developed using a chemiluminescence assay system (Roche) and exposed to Kodak exposure film. Densitometric quantification of the immunoblot data was performed by using Quantity-One software (version 1.2; Bio-Rad Laboratories, Inc.).

Immunofluorescence. Klebsiella pneumoniae cells were prepared as standard operation. Klebsiella pneumoniae cells were fixed with formaldehyde solution (10\%). Rehydrated slides or cells were incubated with primary antibodies: Anti-PBP (dilution 1:500, cat. no. ab226275, Abcam) or anti-zinc finger 
Table I. Primers used for quantitative real-time PCR.

Sequence

\begin{tabular}{|c|c|c|}
\hline Gene name & Reverse & Forward \\
\hline BlaR1 & 5'-TCTAGAGGATCATATTACAATACCGAGCTC-3' & 5'-GAGCTCGGTATTGTAATATGATCCTCTAGA-3' \\
\hline Blal & 5'-CGCTTAATTCAGCACTAAAC-3' & 5'-GAGCTCGGTATTGTAATATGATCCTCTAGA-3' \\
\hline$\beta$-actin & 5'-CGGAGTCAACGGATTTGGTC-3' & 5'-AGCCTTCTCCATGGTCGTGA-3' \\
\hline
\end{tabular}

nuclease (dilution 1:1,000, cat. no, WH0284312M2-100UG; Sigma-Aldrich; Merck KGaA) for $2 \mathrm{~h}$ at $37^{\circ} \mathrm{C}$. Samples were washed with PBS three times and then incubated with anti-IgG-FITC (dilution 1:1,000, cat. no. ab6785; Abcam). Samples were washed and imaged using a fluorescence microscopy (Nikon; magnification, x100).

Electrospray ionization-mass spectrometric analysis. The mass spectrometry was recorded using a quadrupole time-of-flight mass filter (Micromass, UK). Spectrometric analysis was scanned over a range 100-2,000 units of ratio $\mathrm{m} / \mathrm{z}$ of mass to charge, with scan step $2 \mathrm{sec}$ and interscan $0.1 \mathrm{sec} / \mathrm{step}$. The quadrupole scan mode was performed under an electrospray voltage $3 \mathrm{kV}$ at the tip of a stainless-steel capillary needle. The elution conditions were acetonitrile (50\%) containing formic acid $(0.1 \%)$ at rate $2 \mu \mathrm{l} / \mathrm{min}$.

Knockdown gene-coding PBPS. A total of $1 \times 10^{6}$ cells in $100 \mu \mathrm{l}$ $\mathrm{H}_{2} \mathrm{O}$ were transfected with control siRNA negative control (control, $30 \mathrm{nM}$, 5'-UCACAACCUCCUAGAAAGAGU AGA-3') or siRNA targeting PBPs (PBP, $30 \mathrm{nM}, 5$ '-CUGAAG UGAUGUGUAACUGAUCAG-3') using Hiperfect (Qiagen) transfection reagent. After $48 \mathrm{~h}$ of transfection, the transfected cells were treated with zinc finger nuclease $(0.5 \mu \mathrm{g} / \mathrm{ml}$, Sigma-Aldrich; Merck KGaA) for $12 \mathrm{~h}$ at $37^{\circ} \mathrm{C}$.

Statistical methods. All presented data are reported as means \pm SEM. Unpaired data were analyzed by Student's t-test. Comparisons of data between multiple groups were analyzed by analysis of variance (ANOVA) followed by Tukey post hoc test. ${ }^{*} \mathrm{P}<0.05$ and $^{* * *} \mathrm{P}<0.01$ were assigned to indicate statistical significance and are denoted with the relevant asterisks in the figures.

\section{Results}

Identification of ESBL gene expression and antimicrobial drug resistance of Klebsiella pneumoniae. In order to analysis the antimicrobial drug resistance of Klebsiella pneumoniae for $\beta$-lactam, we first determined ESBL gene expression and evaluated $\beta$-lactamase activity. The results in Fig. 1A demonstrate that both the mRNA and protein expression levels of ESBL were higher in Klebsiella pneumoniae isolated from patients with pneumonia (PD-K.p) than natural being Klebsiella pneumonia (NB-K.p). A band at $42 \mathrm{kDa}$ protein was identified in the cell extract of Klebsiella pneumoniae. To determine the role of ESBL in the antimicrobial resistance of Klebsiella pneumoniae, the growth of Klebsiella pneumoniae was assessed.
Our data showed that $\beta$-lactamase could hydrolyze the $\beta$-lactam ring, as determined by electrospray ionization-mass spectrometric analysis (Fig. 1B). PD-K.p presented a higher survival than NB-K.p in an environment with penicillin-based antibiotics (Fig. 1C). This may be due to the higher production of $\beta$-lactamase in PD-K.p than NB-K.p To determine the $\beta$-lactamase activity in vivo, ampicillin was utilized as a substrate to confirm the activity of $\beta$-lactamase. The data in Fig. 1D illustrate that new signals with $\mathrm{m} / \mathrm{z} 368$ units $\mathrm{M}-\mathrm{H}^{+}$ and 390 units $\mathrm{M}-\mathrm{Na}^{+}$were observed in NB-K.p after incubation with $\beta$-lactamase protein at $25^{\circ} \mathrm{C}$ for $30 \mathrm{~min}(\mathrm{~m} / \mathrm{z}$ of ampicillin is 150 units $\mathrm{M}-\mathrm{H}^{+}$and 172 units $\mathrm{M}-\mathrm{Na}^{+}$of PD-K.p). These data suggest that $\beta$-lactamase hydrolyzed the amide bond of the $\beta$-lactam ring and promoted antimicrobial drug resistance of PD-K.p.

Biological functions of PBPs in Klebsiella pneumoniae growth. Penicillin-binding proteins (PBPs), bacterial peripheral membrane enzymes, play an essential role in catalysis in the final steps for the biosynthesis of the essential bacterial cell wall peptidoglycan. PBPs are involved in antimicrobial drug resistance In this study, we aimed to ascertain whether PBPs $(25 \mathrm{mg} / \mathrm{ml})$ could attenuate antimicrobial drug resistance of Klebsiella pneumoniae for $\beta$-lactam antibiotics. Our results showed that PBPs could bind with $\beta$-lactam antibiotic (penicillin) in vitro (Fig. 2A). It was also found that PBPs inhibited Klebsiella pneumoniae growth (PD-K.p and NB-K.p) compared to the control after incubation with penicillin (Fig. 2B). This revealed that PBPs significantly inhibited the growth of PD-K.p compared to NB-K.p (P<0.01 vs. NB-K.p). In addition, our data in Fig. $2 \mathrm{C}$ showed that penicillin could bind the FITC-labeled PBP-specific antibody, and these results suggested that PBPs were expressed and presented at the cell surface of Klebsiella pneumonia, as determined by confocal fluorescence microscope. Furthermore, PBPs downregulated expression of $\beta$-lactamase (Fig. 2D). These results suggest that the binding of PBPs to $\beta$-lactam may interfere with the synthesis of $\beta$-lactamases.

Efficacy of zinc finger nuclease for growth of Klebsiella pneumoniae. A previous study indicates that zinc finger nuclease technology is able to target and disrupt gene-encoded $\beta$-lactamase, which prevents horizontal gene transfer-mediated evolution of antibiotic resistant bacteria and antibiotic resistance genes (23). Therefore, we analyzed the function of zinc finger nuclease in Klebsiella pneumoniae. Fig. 3A shows the antimicrobial susceptibility profile of Klebsiella pneumoniae. The results revealed that Klebsiella pneumoniae 
A
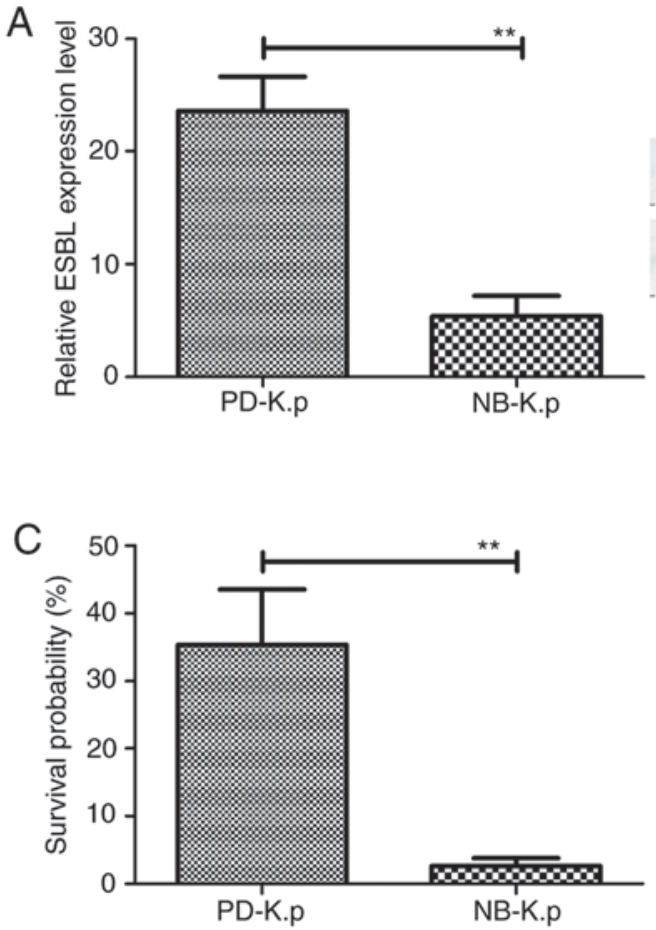

B

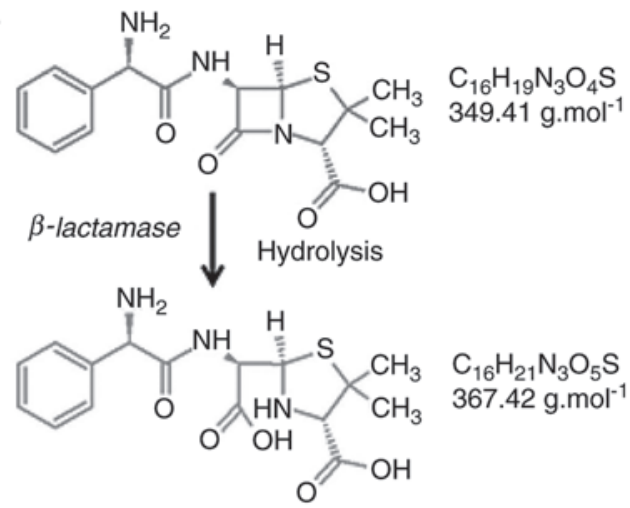

D

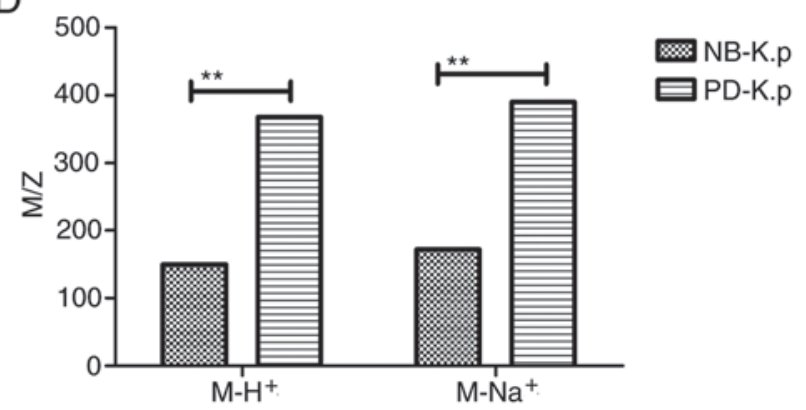

Figure 1. ESBL expression in Klebsiella pneumoniae isolated from a patient with pneumonia. (A) ESBL mRNA and protein expression levels were analyzed in NB-K.p and PD-K.p (B) Detection of the decomposition product by $\beta$-lactamase. (C) $\beta$-lactamase allowed PD-K.p to survive in an environment with penicillin-based antibiotics. (D) M/Z values as analyzed in NB-K.p and PD-K.p for penicillin. Data are presented as the mean \pm SEM. ${ }^{* *} \mathrm{P}<0.01$, statistically significant difference. NB-K.p, natural being Klebsiella pneumoniae; PD-K.p, Klebsiella pneumoniae from a patient with pneumonia; ESBL, extended-spectrum $\beta$-lactamase.
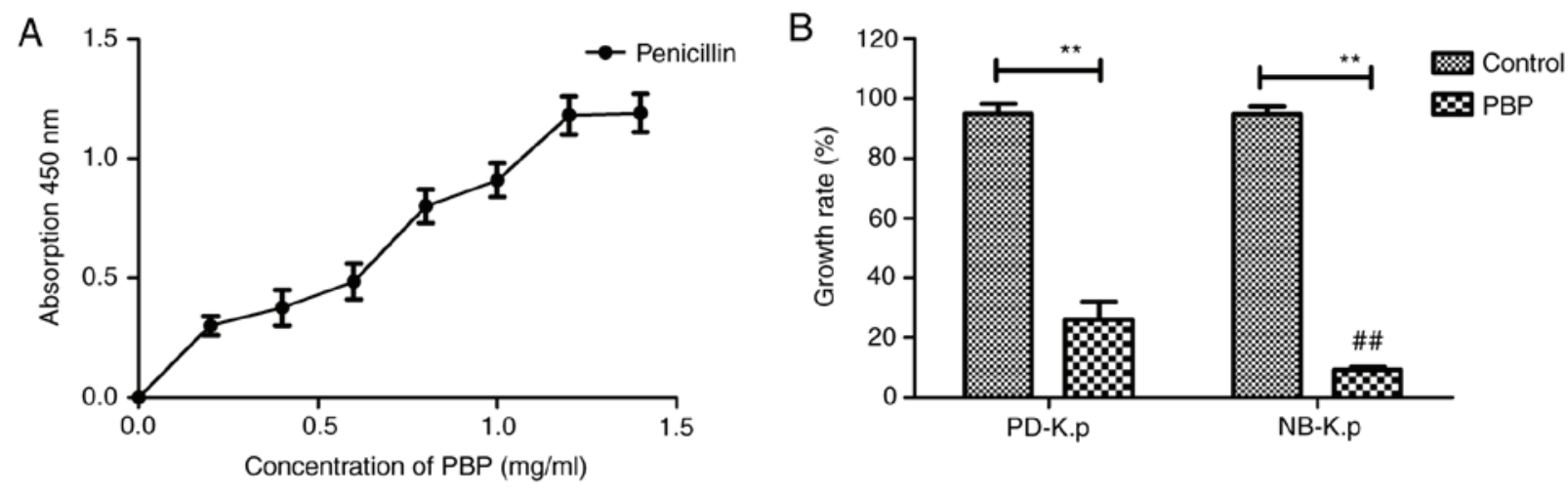

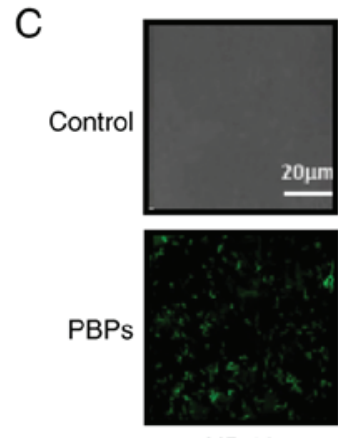

NB-K.p
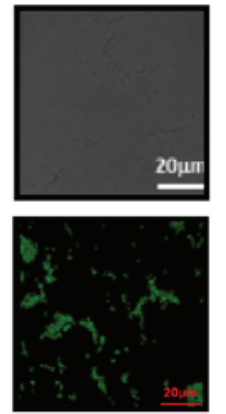

PD-K.p

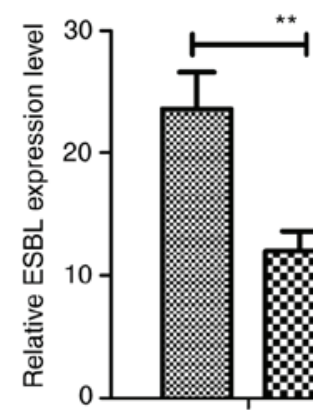

PD-K.p
Control

$\infty$ PBP

Figure 2. Biological functions of PBPs in regards to Klebsiella pneumoniae growth. (A) ELISA was used to analyze the affinity between PBPs and $\beta$-lactam antibiotic (penicillin) in vitro. (B) PBP efficiently inhibited the growth of NB-K.p and PD-K.p at a concentration of $25 \mathrm{mg} / \mathrm{ml}$. (C) PBP expression was located on the cell surface of Klebsiella pneumonia as determined by confocal fluorescence microscope. (D) PBP treatment downregulated expression of $\beta$-lactamases. Data are presented as the mean \pm SEM. ${ }^{*} \mathrm{P}<0.05,{ }^{* *} \mathrm{P}<0.01$, statistically significant difference. NB-K.p, natural being Klebsiella pneumoniae; PD-K.p, Klebsiella pneumoniae from a patient with pneumonia; ESBL, extended-spectrum $\beta$-lactamase; PBPs, penicillin-binding proteins. 

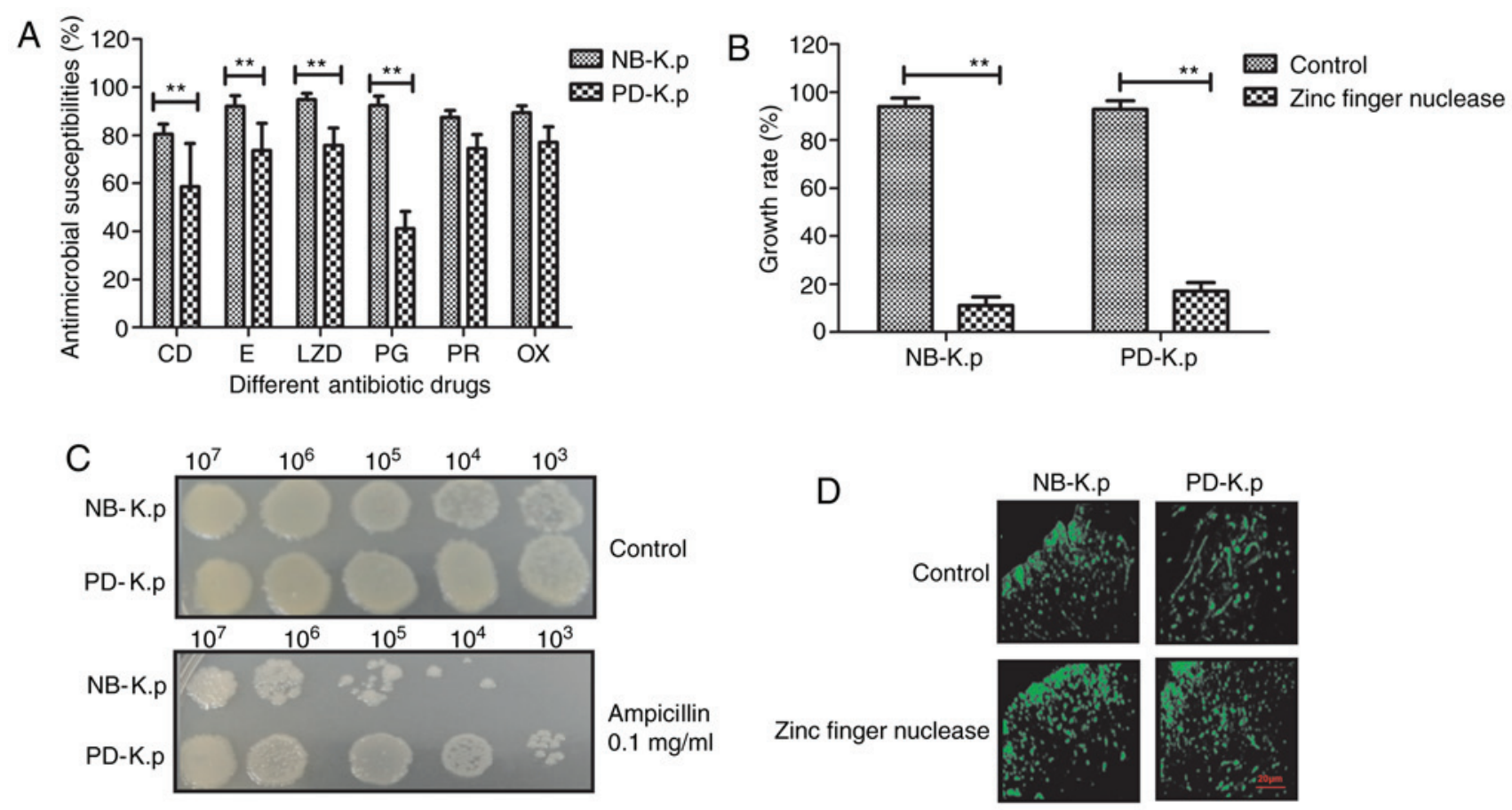

Figure 3. Efficacy of zinc finger nuclease on the growth of Klebsiella pneumoniae. (A) Antimicrobial susceptibility profile of Klebsiella pneumoniae was analyzed. (B) Zinc finger nuclease treatment attenuated the drug resistance of Klebsiella pneumoniae when compared to the control. (C) The optical density experiment demonstrated a marked decrease in Klebsiella pneumoniae growth after treatment with ampicillin compared with the control groups. (D) Zinc finger nuclease significantly increased the binding to penicillin as compared with that control. Data are presented as the mean \pm SEM. ${ }^{* *} \mathrm{P}<0.01$, statistically significant difference. CD, clindamycin; E, erythromycin; LZD, linezolid; PG, penicillin G; RP, rifampin; OX, oxacillin; NB-K.p, natural being Klebsiella pneumoniae; PD-K.p, Klebsiella pneumoniae from a patient with pneumonia.

bacteria exhibit varying degrees of multi-drug resistance (CD, clindamycin; E, erythromycin; LZD, linezolid; PG, penicillin G; RP, rifampin; OX, oxacillin; concentrations of all the antibiotics were $1 \mathrm{mg} / \mathrm{ml}$ ). However, zinc finger nuclease treatment decreased drug resistance of Klebsiella pneumoniae compared to control. Fig. 3B indicates that the growth of Klebsiella pneumoniae was inhibited after transduction of plasmids containing zinc finger nuclease. The optical density experiment demonstrated a marked decrease in Klebsiella pneumoniae growth after treatment with ampicillin compared with the control groups $(\mathrm{P}<0.01)$ at the indicated temperature, which was consistent with the results in Fig. 3A (Fig. 3C). In addition, the kinetics of zinc finger nuclease in Klebsiella pneumoniae was analyzed. The data showed that zinc finger nuclease markedly increased the binding potential of PD-K.p to penicillin compared with PBS (control) (Fig. 3D). The data suggest that zinc finger nuclease inhibited Klebsiella pneumoniae growth and enhanced the binding capacity of penicillin to Klebsiella pneumoniae.

Relationship among $\beta$-lactamase, PBPs and zinc finger nuclease in PD-K.p. To understand the mechanism of zinc finger nuclease in inhibiting Klebsiella pneumoniae growth, we analyzed the relationship among $\beta$-lactamase, PBPs and zinc finger nuclease. First, we detected expression levels of $\beta$-lactamase and PBPs in Klebsiella pneumoniae after incubation with zinc finger nuclease for $24 \mathrm{~h}$. Fig. 4A shows that zinc finger nuclease treatment upregulated PBP expression and downregulated $\beta$-lactamase production. Knockdown of gene-coding PBPs abrogated the inhibitory effects on zinc finger nuclease for PD-K.p (Fig. 4B). Importantly, our data showed that the inhibitory effects of zinc finger nuclease were more superior to PBPs at the same concentration $(10 \mathrm{mg} / \mathrm{ml})$ in regards to PD-K.p growth (Fig. 4C). Notably, expression levels of two members (BlaR1 and Blal) of the $\beta$-lactamase signaling pathway in PD-K.p were decreased after zinc finger nuclease treatment (Fig. 4D). To discover the action site of zinc finger nuclease, we transfected plasmids of rpGFP-ZFN into Klebsiella pneumoniae with rpGFP as control. As shown in Fig. 4E, strong fluorescence was observed in surrounding Klebsiella pneumoniae cells delivering rpGFP-ZFN plasmid, while fluorescence was found in the cytoplasm carrying rpGFP, indicating that the action site of zinc finger nuclease is associated with the membrane of Klebsiella pneumoniae. Fig. 4F further confirmed that the action site of zinc finger nuclease is located in the outer membrane of Klebsiella pneumoniae. These results indicate that zinc finger nuclease could regulate PBPs or zinc finger nuclease expression, suggesting that it may represent a potential agent for antibiotic resistance.

\section{Discussion}

The mean number of gram-positive bacilli isolated from hospitalized patients has gradually revealed the different degree of antibiotic resistance since 2000 (26,27). Resistance to antimicrobial drugs is one of the greatest challenges due to the overuse and misuse of antibiotics in modern medicine, which significantly threatens public health $(28,29)$. In the present study, the potential molecular mechanism of antibiotic resistance of 

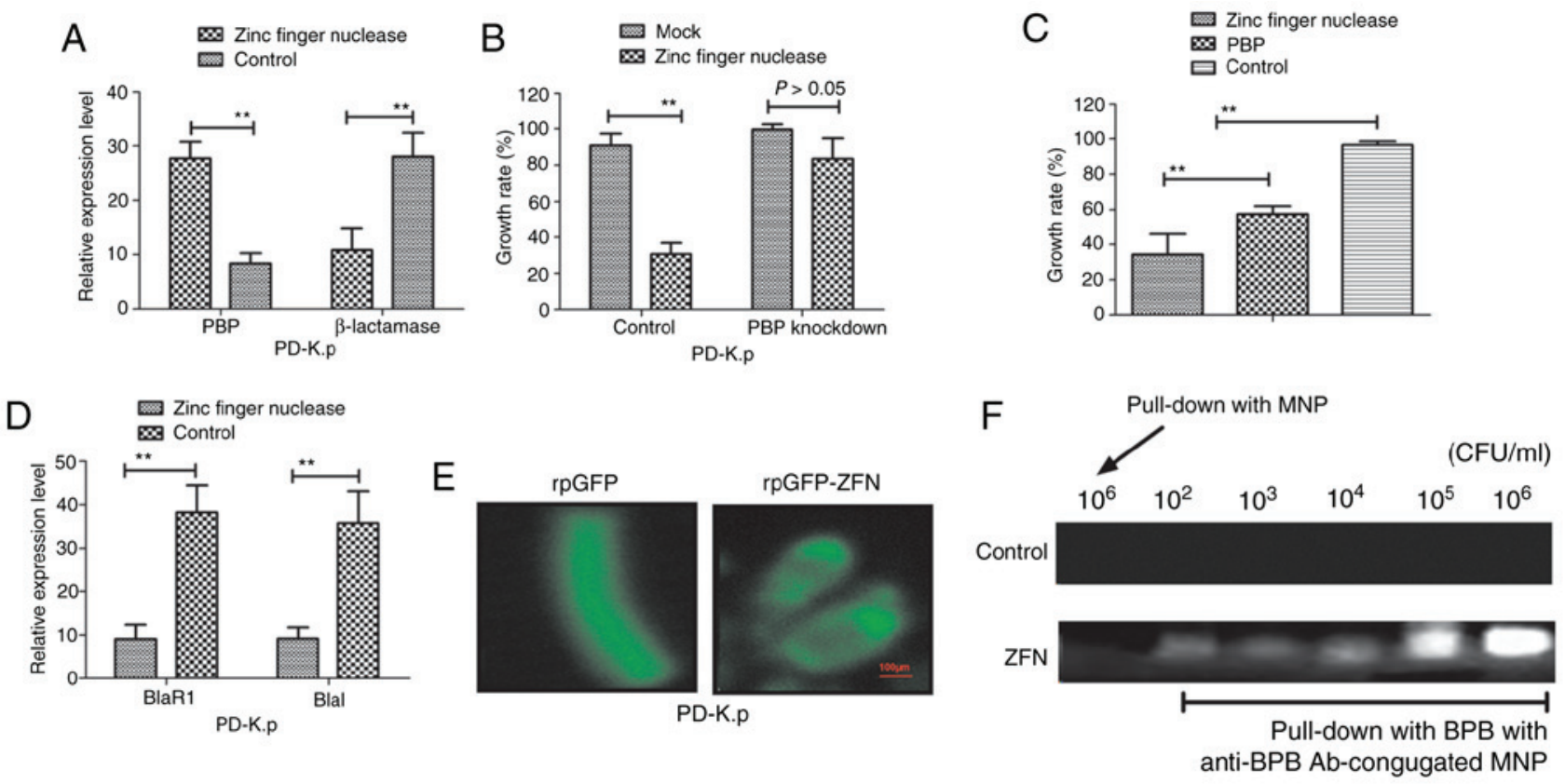

Figure 4. Analysis of the association among $\beta$-lactamase, PBPs and zinc finger nuclease in PD-K.p (A) mRNA expression levels of PBP and $\beta$-lactamase were analyzed in PD-K.p (B) PBP knockdown suppressed the inhibitory effects of zinc finger nuclease on PD-K.p growth. (C) Zinc finger nuclease was superior to PBP in inhibiting PD-K.p growth. (D) Zinc finger nuclease inhibited BlaR1 and Blal expression in the $\beta$-lactamase signaling pathway in PD-K.p (E) Fluorescence microscopic detection of the action site of zinc finger nuclease in PD-K.p (F) Upper panel shows the analysis of the immunoprecipitation of zinc finger nuclease (ZFN) in PD-K.p cells with antibody-conjugated magnetic nanoparticles (MNPs). Data are presented as the mean \pm SEM. ${ }^{* *} \mathrm{P}<0.01$, statistically significant difference. NB-K.p, natural being Klebsiella pneumoniae; PD-K.p, Klebsiella pneumoniae from a patient with pneumonia; PBPs, penicillin-binding proteins.

Klebsiella pneumonia was investigated. Although a previous study has proposed a primary theory of antibiotic resistance, the main viewpoint focuses on the $\beta$-lactamase signaling (30). In the present study, extended-spectrum $\beta$-lactamase produced by Klebsiella pneumoniae was confirmed by susceptibility testing. Notably, our data indicated that penicillin-binding proteins (PBPs) enhanced the affinity between Klebsiella pneumoniae and penicillin, which led to the inhibition of the growth of Klebsiella pneumoniae. Importantly, zinc finger nuclease targeting the $\beta$-lactam resistance gene led to the downregulation of $\beta$-lactamase synthesis. We also found that expression of PBPs was decreased in Klebsiella pneumoniae after treatment with zinc finger nuclease. We concluded that zinc finger nuclease not only regulated the biological activity of $\beta$-lactamase, but also promoted expression of PBPs in Klebsiella pneumoniae.

Antibiotic resistance in Klebsiella pneumoniae is associated with high rates of morbidity and mortality in clinical patients (31). Therefore, understanding the mechanisms involved in the antibiotic resistance of Klebsiella pneumoniae are highly required to solve the antibiotic resistance problem and decrease mortality of patients infected with Klebsiella pneumoniae. $\beta$-lactam antibiotic resistance is mainly caused by two mechanisms including extended-spectrum $\beta$-lactamase (ESBL) and target mutation of gene-coding PBPs $(32,33)$. The results of the present study found that ESBL expression was upregulated by the indiscriminate application of antibiotics, which plays a hydrolytic role in $\beta$-lactam antibiotic resistance. The expression level of $\beta$-lactamases is responsible for $\beta$-lactam resistance in Klebsiella pneumoniae by catalyzing the hydrolysis of $\beta$-lactam antibiotics to generate antimicrobiologically inactive compounds (34). In addition, a recent study indicated that the genome of the $\beta$-lactamase superfamily including RNA-metabolizing metallo- $\beta$-lactamases and $\beta$-lactamase domain-containing proteins are expanded in antibiotic-resistance Klebsiella pneumoniae (35). Furthermore, a study has showed that various types of $\beta$-lactamases are distributed in a variety of organisms (36). In the present study, it was observed that the antibiotic resistance of Klebsiella pneumoniae was associated with $\beta$-lactamase expression (37). ESBL-producing bacteria are resistant to most $\beta$-lactam antibiotics (38). ESBL-producing Klebsiella pneumoniae with genetic diversity is characterized with resistance genes, which is a significant threat for public health (39). This study found that patient-derived PD-K.p produced more ESBL than natural being NB-K.p, which contributed to the increasing of drug resistance. Future study should be specifically targeted towards the ESBL signaling pathway as it is crucial to guide antibiotic therapy for patients infected with PD-K.p.

A previous study has suggested that zinc finger nuclease may be a potential agent to overcome $\beta$-lactam antibiotic resistance (23). Antibiotic resistance of Klebsiella pneumoniae has challenged the therapeutic efficacy for patients with pneumonia (35). This preclinical study described the efficacy of zinc finger nuclease technology via targeting and disrupting the high expression of lactamase in Klebsiella pneumoniae, which prevented horizontal gene transfer-mediated antibiotic resistance. Our data showed that zinc finger nuclease targeting the $\beta$-lactam resistance gene led to downregulation of the $\beta$-lactamase signaling pathway and decreased $\beta$-lactamase 
synthesis and therefore attenuated $\beta$-lactam resistance. The results also found that zinc finger nuclease promoted PBP synthesis, which enhanced the inhibitory effects of $\beta$-lactam on the growth of Klebsiella pneumoniae. However, the present study only analyzed the role of PBP synthesis in Klebsiella pneumoniae growth, and further assessment of the function of PBPs need further investigation. In addition, development of an advanced zinc finger nuclease archive to target a broader range of DNA sequences would allow zinc finger nuclease to target the catalytic domain encoding region of drug resistance, which may result in more efficient disruption of resistance. Furthermore, increasing zinc finger nuclease expression may be a potential strategy to reduce antibiotic resistance in Klebsiella pneumoniae.

In conclusion, the present study elucidated the molecular mechanisms of the $\beta$-lactam resistance of Klebsiella pneumoniae. PBPs and zinc finger nuclease were utilized to design the drug resistance, while zinc finger nuclease was found to be a potent regulator of PBP expression. Consistent with a previous study, $\beta$-lactamases in antibiotic-resistance Klebsiella pneumonia are various and $\mathrm{PBP}$ expression is variable in the $\beta$-lactam system (40). In addition, PBPs and $\beta$-lactamases are two important resistance mechanisms in Klebsiella pneumoniae caused by the frequent use of antibiotics in patients with pneumonia (41). Furthermore, the rate of emergence of new antibiotic-resistant bacteria is rapidly increasing, that leads to an increase in the number of patients presenting with tolerant Klebsiella pneumonia. Taken together, the resistance of Klebsiella pneumoniae bacteria to antimicrobial drugs acts via the ESBL signaling pathway, which may be a potential target for the treatment of patients infected with Klebsiella pneumoniae through the $\beta$-lactamase signaling pathway.

\section{Acknowledgements}

Not applicable.

\section{Funding}

This study was supported by a grant from the National Science Foundation (China).

\section{Availability of data and materials}

The datasets used and/or analyzed during the current study are available from the corresponding author on reasonable request.

\section{Authors' contributions}

WY performed experiments and data analysis. XZ, NW and HR performed experiments, collected data and prepared the diagrams. WJ designed the experiments and wrote the manuscript.

\section{Ethics approval and consent to participate}

This study was approved by the Ethics Committee of Tianjin First Central Hospital (Tianjin, China). The patient enrolled in this study signed a written consent form before participation.

\section{Patient consent for publication}

Not applicable.

\section{Competing interests}

The authors declare that they have no competing interests.

\section{References}

1. Lim MK, Lai PS, Ponnampalavanar SS, Syed Omar SF, Taib NA, Yusof MY, Italiano CM, Kong DC and Kamarulzaman A: Antibiotics in surgical wards: Use or misuse? A newly industrialized country's perspective. J Infect Dev Countries 9: 1264-1271, 2015.

2. Kardas P, Devine S, Golembesky A and Roberts C: A systematic review and meta-analysis of misuse of antibiotic therapies in the community. Int J Antimicrob Agents 26: 106-113, 2005.

3. Heineman HS and Watt VS: All-inclusive concurrent antibiotic usage review: A way to reduce misuse without formal controls. Infect Control 7: 168-171, 1986.

4. Nõlvak H, Truu M, Kanger K, Tampere M, Espenberg M, Loit E, Raave $\mathrm{H}$ and Truu J: Inorganic and organic fertilizers impact the abundance and proportion of antibiotic resistance and integron-integrase genes in agricultural grassland soil. Sci Total Environ 562: 678-689, 2016.

5. Stogios PJ, Cox G, Spanogiannopoulos P, Pillon MC, Waglechner N, Skarina T, Koteva K, Guarné A, Savchenko A and Wright GD: Rifampin phosphotransferase is an unusual antibiotic resistance kinase. Nat Commun 7: 11343, 2016.

6. Ferro G, Guarino F, Castiglione S and Rizzo L: Antibiotic resistance spread potential in urban wastewater effluents disinfected by $\mathrm{UV} / \mathrm{H}_{2} \mathrm{O}_{2}$ process. Sci Total Environ 560-561: 29-35, 2016.

7. Ouwehand AC, Forssten S, Hibberd AA, Lyra A and Stahl B: Probiotic approach to prevent antibiotic resistance. Ann Med 48: 246-255, 2016.

8. Date KA, Newton AE, Medalla F, Blackstock A, Richardson L, McCullough A, Mintz ED and Mahon BE: Changing patterns in enteric fever incidence and increasing antibiotic resistance of enteric fever isolates in the United States, 2008-2012. Clin Infect Dis 63: 322-329, 2016.

9. Çöl A, Dedeić-Ljubović A, Salimović-Bešić I and Hukic M: Antibiotic resistance profiles and genetic similarities within a new generation of carbapenem-resistant Acinetobacter calcoaceticus-A. baumannii complex resistotypes in Bosnia and Herzegovina. Microb Drug Resist 22: 655-661, 2016.

10. Barger A, Schaade L, Krause G and Kramer M: Strategies for recognition, prevention and control of antimicrobial resistances in Germany-a draft from the federal german ministry of health. Gesundheitswesen 70: 631-635, 2008 (In German).

11. Oliva A, Gizzi F, Mascellino MT, Cipolla A, D'Abramo A, D'Agostino C, Trinchieri V, Russo G, Tierno F, Iannetta M, et al: Bactericidal and synergistic activity of double-carbapenem regimen for infections caused by carbapenemase-producing Klebsiella pneumoniae. Clin Microbiol Infect 22: 147-153, 2016.

12. Gajul SV, Mohite ST, Mangalgi SS, Wavare SM and Kakade SV: Klebsiella pneumoniae in septicemic neonates with special reference to extended spectrum $\beta$-lactamase, AmpC, metallo $\beta$-lactamase production and multiple drug resistance in tertiary care hospital. J Lab Physicians 7: 32-37, 2015.

13. Rastogi V, Nirwan PS, Jain S and Kapil A: Nosocomial outbreak of septicaemia in neonatal intensive care unit due to extended spectrum $\beta$-lactamase producing Klebsiella pneumoniae showing multiple mechanisms of drug resistance. Indian J Med Microbiol 28: 380-384, 2010.

14. Babálová M, Blahová J, Králiková K, Kreméry V Sr, Menkyna R, Bartoníková N and Skalicková R: Unexpected reservoir of Klebsiella pneumoniae strains with transferable multiple drug resistance causing clinical sepsis in newborns. J Chemother 17: 454-455, 2005.

15. Sanchez GV, Master RN, Clark RB, Fyyaz M, Duvvuri P, Ekta G and Bordon J: Klebsiellapneumoniae antimicrobial drug resistance, United States, 1998-2010. Emerg Infect Dis 19: 133-136, 2013. 
16. Neupane S, Pant ND, Khatiwada S, Chaudhary R and Banjara MR: Correlation between biofilm formation and resistance toward different commonly used antibiotics along with extended spectrum beta lactamase production in uropathogenic Escherichia coli isolated from the patients suspected of urinary tract infections visiting Shree Birendra Hospital, Chhauni, Kathmandu, Nepal. Antimicrob Resist Infect Control 5: 5, 2016.

17. Chen X, Che J, Zhao XF, Zhang LF and Li J: Dissemination of insertion sequence common regions 1 and int 1 gene and drug resistance of 483 Escherichia coli and Klebsiella pneumonia broiler isolates. Zhonghua Yu Fang Yi Xue Za Zhi 51: 886-889, 2017 (In Chinese).

18. Sekowska A, Janicka G, Klyszejko C, Wojda M, Wroblewski M and Szymankiewicz M: Resistance of Klebsiella pneumoniae strains producing and not producing ESBL (extended-spectrum beta-lactamase) type enzymes to selected non-beta-lactam antibiotics. Med Sci Monit 8: BR100-BR104, 2002.

19. Sanguinetti CM, De Benedetto F and Miragliotta G: Bacterial agents of lower respiratory tract infections (LRTIs), beta-lactamase production, and resistance to antibiotics in elderly people. DEDALO study group. Int J Antimicrob Agents 16: 467-471, 2000.

20. Boggio SB and Roveri OA: Catalytic properties of an endogenous beta-lactamase responsible for the resistance of Azospirillum lipoferum to beta-lactam antibiotics. Microbiology 149: 445-450, 2003.

21. Uraz G, Simsek H and Celik B: Beta-Lactamase activities and resistance to antibiotics of Haemophilus influenzae, $\mathrm{H}$. parainfluenzae and $\mathrm{H}$. aphrophilus strains identified in throat cultures from children. Drug Metabol Drug Interact 16: 217-228, 2000

22. Macheboeuf P, Contreras-Martel C, Job V, Dideberg O and Dessen A: Penicillin binding proteins: Key players in bacterial cell cycle and drug resistance processes. FEMS Microbiol Rev 30: 673-691, 2006.

23. Shahbazi Dastjerdeh M, Kouhpayeh S, Sabzehei F, Khanahmad H, Salehi M, Mohammadi Z, Shariati L, Hejazi Z, Rabiei P and Manian M: Zinc finger nuclease: A new approach to overcome beta-lactam antibiotic resistance. Jundishapur J Microbiol 9: e29384, 2016.

24. Zscheppang K, Kurth I, Wachtel N, Dubrovska A, KunzSchughart LA and Cordes N: Efficacy of betal integrin and EGFR targeting in sphere-forming human head and neck cancer cells. J Cancer 7: 736-745, 2016.

25. Hsueh PR, Ko WC, Wu JJ, Lu JJ, Wang FD, Wu HY, Wu TL and Teng LJ: Consensus statement on the adherence to clinical and laboratory standards institute (CLSI) antimicrobial susceptibility testing guidelines (CLSI-2010 and CLSI-2010-update) for enterobacteriaceae in clinical microbiology laboratories in Taiwan. J Microbiol Immunol Infect 43: 452-455, 2010.

26. Baruah FK, Hussain AN, Kausalya and Grover RK: Antibiotic resistance profile of non-fermenting Gram-negative bacilli isolated from the blood cultures of cancer patients. J Glob Infect Dis 7: 46-47, 2015

27. Hill JT, Tran KD, Barton KL, Labreche MJ and Sharp SE: Evaluation of the nanosphere Verigene BC-GN assay for direct identification of gram-negative bacilli and antibiotic resistance markers from positive blood cultures and potential impact for more-rapid antibiotic interventions. J Clin Microbiol 52: 3805-3807, 2014.

28. Ares MA, Alcantar-Curiel MD, Jimenez-Galicia C, Rios-Sarabia N, Pacheco S and De la Cruz MA: Antibiotic resistance of gram-negative bacilli isolated from pediatric patients with nosocomial bloodstream infections in a mexican tertiary care hospital. Chemotherapy 59: 361-368, 2013.
29. Patel SJ, O'Toole D and Larson E: A new metric of antibiotic class resistance in gram-negative bacilli isolated from hospitalized children. Infect Control Hosp Epidemiol 33: 602-607, 2012.

30. Sasirekha B and Shivakumar S: Occurrence of plasmid-mediated AmpC $\beta$-lactamases among Escherichia coli and Klebsiella pneumoniae clinical isolates in a tertiary care hospital in Bangalore. Indian J Microbiol 52: 174-179, 2012.

31. Koczura R, Mokracka J, Krzyminska S and Kaznowski A: Virulence properties and integron-associated antibiotic resistance of Klebsiella mobilis strains isolated from clinical specimens. J Med Microbiol 60: 281-288, 2011.

32. Lee M, Dhar S, De Benedetti S, Hesek D, Boggess B, Blázquez B, Mathee $\mathrm{K}$ and Mobashery S: Muropeptides in pseudomonas aeruginosa and their role as elicitors of $\beta$-lactam-antibiotic resistance. Angew Chem Int Ed Engl 55: 6882-6886, 2016.

33. Lee YD and Park JH: Phage conversion for $\beta$-lactam antibiotic resistance of staphylococcus aureus from foods. J Microbiol Biotechnol 26: 263-269, 2016.

34. Shafiq M, Rahman H, Qasim M, Ayub N, Hussain S, Khan J and Naeem M: Prevalence of plasmid-mediated AmpC $\beta$-lactamases in Escherichia coli and Klebsiella pneumonia at tertiary care hospital of Islamabad, Pakistan. Eur J Microbiol Immunol (Bp) 3: 267-271, 2013.

35. Goessens WH, Mouton JW, Ten Kate MT, Sorgel F, Kinzig M and Bakker-Woudenberg IA: The therapeutic effect of tigecycline, unlike that of Ceftazidime, is not influenced by whether the Klebsiella pneumoniae strain produces extended-spectrum beta-lactamases in experimental pneumonia in rats. Antimicrob Agents Chemother 57: 643-646, 2013.

36. Rivera A, Larrosa N, Mirelis B and Navarro F: Importance of quality control for the detection of $\beta$-lactam antibiotic resistance in enterobacteriaceae. Enferm Infecc Microbiol Clin 32 (Suppl 1): S30-S36, 2014 (In Spanish).

37. Padilla E, Alonso D, Domenech-Sanchez A, Gomez C, Pérez JL, Albertí S and Borrell N: Effect of porins and plasmid-mediated AmpC beta-lactamases on the efficacy of beta-lactams in rat pneumonia caused by Klebsiella pneumoniae. Antimicrob Agents Chemother 50: 2258-2260, 2006.

38. Wragg R, Harris A, Patel M, Robb A, Chandran $\mathrm{H}$ and McCarthy L: Extended spectrum beta lactamase (ESBL) producing bacteria urinary tract infections and complex pediatric urology. J Pediatr Surg 52: 286-288, 2017.

39. Charrouf FO, Hamze M, Mallat H, Achkar M and Dabboussia F: Characterization of resistance genes in 68 ESBL-producing Klebsiella pneumonia in Lebanon. Med Mal Infect 44: 535-538, 2014.

40. Ogawara H: Self-resistance in streptomyces, with special reference to beta-lactam antibiotics. Molecules 21: E605, 2016.

41. Navarro Jimenez C, Luque Marquez R, de Torres Solis I and Aguilar Guisado M: Changes in empiric therapy for nosocomial pneumonia due to the emergence of bacterial strains with broad spectrum beta-lactamases. Med Clin (Barc) 122: 679, 2004 (In Spanish).

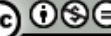

This work is licensed under a Creative Commons Attribution-NonCommercial-NoDerivatives 4.0 International (CC BY-NC-ND 4.0) License. 